\title{
Pharmacokinetic Bioequivalence Study of Two Formulations of Teriflunomide in Healthy Male Volunteers under Fasting Conditions
}

\author{
Sevinsky $E^{1 *}$, Silvestro $L^{2}$, Neatu $A^{2}$, Roffé $C^{3}$ and Rizea-Savu $\mathbf{S}^{2}$ \\ ${ }^{1}$ Clinical Operations and Pharmacovigilance Representative, TUTEUR S.A.C.I.F.I.A, Argentina \\ 23S-Pharmacological Consultation \& Research $\mathrm{GmbH}$, Harpstedt, Germany \\ ${ }^{3}$ Medical Director TUTEUR S.A.C.I.F.I.A.
}

\begin{abstract}
A generic formula containing $14 \mathrm{mg}$ of teriflunomide (Terflimida ${ }^{\circledR}$ test formulation) in oral coated tablets was compared to the reference product $\left(\right.$ Aubagio ${ }^{\circledR}$ ) in a pharmacokinetic, open label, two-periods, two sequences, two-way crossover; block randomized single dose study in healthy male volunteers under fasting conditions. The statistical analysis of the pharmacokinetic data obtained in this study showed that the Teriflunomide formulations: Terflimida $^{\circledR}$ coated tablets 14 (test formulation) and Aubagio ${ }^{\circledR} 14 \mathrm{mg}$ coated tablets (reference formulation), were bioequivalent regarding the rate $\left(C_{\max }\right)$ and the extent of absorption (AUC0-72), under fasting conditions. The Teriflunomide treatments (TEST and REFERENCE formulations), administered orally in single dose, to male healthy volunteers, under fasting conditions, were very well tolerated by all the participating subjects.
\end{abstract}

Keywords: Teriflunomide; Multiple sclerosis; Bioequivalence

\section{Introduction}

Multiple sclerosis (MS) is an immune-mediated and neurodegenerative disease with an unpredictable outcome. Immunemodulatory treatment aims at decreasing long-term disability. MS is categorized into 4 distinct types, primarily based on its clinical course, which are characterized by increasing severity: (a) Relapsing/ remitting MS (RRMS), the most common form, affecting $85 \%$ of all MS patients which involves relapses followed by remission; (b) secondary progressive MS (SPMS), which develops over time following diagnosis of RRMS; (c) primary progressive MS (PPMS) affecting $8-10 \%$ of patients, noted as gradual continuous neurologic deterioration; and (d) progressive relapsing MS (PRMS) the least common form $(<5 \%)$, which is similar to PPMS but with overlapping relapses [1-3]. MS leads to a wide range of symptoms involving different parts of the body. MS symptoms and disease progression are varied, with some individuals experiencing little disability while most (up to 60\%) require a wheelchair 20 year from diagnosis [1].

The treatment of MS has been a challenge with treatment options being limited mainly to corticosteroids, the potent alkylating agent cyclophosphamide and potent immunosuppressant methotrexate. However, with the advent of immunomodulatory drugs in mid1990s, a big shift was carried to treatment options for the first time [4]. All immunotherapeutic drugs target relapsing remitting MS (RRMS) but it still remains a medical challenge in MS to develop a treatment for progressive forms. Until the approval of the first oral treatment in 2010 [3], all MS treatments consisted of either intramuscular or subcutaneous injectable drugs which are related to a lack of compliance. To date, 13 FDA approved disease-modifying drugs are available for RRMS, and several more agents are in different developmental stages [1,3,5,6].

Teriflunomide is an active metabolite of leflunomide (an immunosuppressive disease-modifying drug used for rheumatoid arthritis) which inhibits the dihydroorotate dehydrogenase enzyme and inhibits the proliferation of $\mathrm{B}$ and $\mathrm{T}$ cells [7]. In addition, teriflunomide exerts anti-inflammatory properties by inhibiting IFNgamma producing T cells while IL- 4 and IL- 10 producing T cells are unaffected [8]. In MS, oral administration of teriflunomide reduced relapse rates, MS lesions and decreased disability progression [9-13].
Nowadays, generic formulations play an important role in the accessibility of the drug, especially because they optimized the costbenefit relationship in favor of the patient, his/her family group and social security insurance. The performance of a bioequivalence study for Teriflunomide is not a requirement for the registration of this drug in Argentina. To introduce a similar formulation in the market for this drug only Good Manufacture Practice, quality controls and in vitro dissolution tests results are required: Based on these rules Tuteur S.A.C.I.F.I.A. Laboratory got the approval to commercialize Terflimida, a coated tablet containing $14 \mathrm{mg}$ of teriflunomide for the treatment of MS, on August 26 ${ }^{\text {th }}, 2016$ [14]. However, since it is well known that a similar in vitro result does not always means that the same plasma profile is obtained when analyzed in vivo and also that there is no other available generic formulation for teriflunomide, Tuteur S.A.C.I.F.I.A. Laboratory decided to carry out a formal bioequivalence study following FDA draft Guidance on Terflunomide in order to be able to assure that Aubagio (innovator product) and Terflimida could be interchangeable. Keeping in consideration the long half-life of this product a truncated AUC, from 0 to 72 hours, evaluation was preferred for bioequivalence assessment, in accordance with the above-mentioned FDA rules, while in other published studies AUC0-t was preferred as bioequivalence parameter [15-17]. It is noteworthy that the application of truncated AUC permitted to employ an accelerated elimination procedure with cholestyramine $8 \mathrm{~g}$ tid or $4 \mathrm{~g}$ tid, or activated charcoal, as recommended by the originator (SANOFI, Winthrop Industry), minimizing the risk of teratogenic effects in the healthy volunteers and shortening significantly the duration of the study.

*Corresponding author: Esther Sevinsky, Clinical Operations and Pharmacovigilance Representative, TUTEUR S.A.C.I.F.I.A, Argentina, Tel: +54 11 57872222; E-mail: esthersevinsky@gmail.com

Received May 16, 2018; Accepted May 31, 2018; Published June 09, 2018

Citation: Sevinsky E, Silvestro L, Neatu A, Roffé C, Rizea-Savu S (2018) Pharmacokinetic Bioequivalence Study of Two Formulations of Teriflunomide in Healthy Male Volunteers under Fasting Conditions. J Bioequiv Availab 10: 43-47. 376. doi: 10.4172/0975-0851.1000376

Copyright: (C) 2018 Sevinsky E, et al. This is an open-access article distributed under the terms of the Creative Commons Attribution License, which permits unrestricted use, distribution, and reproduction in any medium, provided the original author and source are credited. 


\section{Methods}

\section{Subjects}

This study, duly approved by an ethical Committee (National Ethical Committee of Moldavia, Chsinau) recruited 24 healthy, Caucasian male volunteers aged 18 to 51 . The investigator considered the subjects healthy if the clinical evaluation, vital signs, electrocardiogram and clinical laboratory had normal results or showed no clinical relevance. The same investigator also evaluated the subject as reliable taking into account the participation in previous studies. All the subjects included signed an informed consent and showed responsibility, compliance and understanding of what his participation in the study involved. Exclusion criteria were history of hypersensitivity to the test drug or to drugs belonging to the same pharmacological and chemical class or to the inactive ingredients of the formulations, rare hereditary problems of glucose intolerance, the Lapp lactase deficiency, glucose-galactose malabsorption or gluten intolerance, participation in a clinical study with a new investigational product in the preceding three months or in a clinical study with a generic product in the preceding two months, hospitalization for any reason within eight weeks prior to the study initiation, donation of $450 \mathrm{ml}$ or more of blood, within eight weeks prior to the study initiation, evidence or history of cardiovascular, pulmonary, renal, hepatic, pancreatic, hematological, gastrointestinal, immune disorders, endocrine or neoplastic diseases, immunization during previous 4 weeks, any recent history (within the last two years) of drug or alcohol abuse, recent psychiatric disorder or use of psychotropic medicines and history or any current condition or other disease known to interfere with the absorption, distribution, metabolism or excretion of the investigational drug. The study was performed in compliance with ICH Topic E6 Good Clinical Practice (GCP), Consolidated Guidance, including the archiving of essential documents.

\section{Formulations}

The test formulation, Terflimida ${ }^{\circ}$, was a coated tablet containing $14 \mathrm{mg}$ of teriflunomide manufactured by VICROFER S.R.L/TUTEUR S.A.C.I.F.I.A, Buenos Aires (Batch No TF201B) and the reference product, Aubagio, was also a coated tablet containing the same dose of teriflunomide, manufactured by Winthrop Ind., France and imported/ distributed by Genzyme Argentina S.A., Argentina (Batch No. 6LN3D).

\section{Study design}

The clinical trial was an open label, two-period, two-sequence, crossover, controlled, block randomized bioequivalence study on healthy male volunteers, under fasting conditions. Blood samples were taken before (time 0.0 ) and at the following times after study drug administration: $0.5,1.0,1.5,2.0,2.5,3.0,3.5,4.0,4.5,5.0,5.5,6.0,6.5$, $7.0,8.0,10.0,12.0,16.0,20.0,24.0,32.0,48.0,56.0$ and 72.0 hours. The subjects were hospitalized (Clinical Hospital of the Ministry of Health in Chsinau) from the evening prior to the drug administration until 32 hours post dose under medical observation. For the collection of the remaining blood samples (at 48.0, 56.0 and 72.0 hours post dose) the subjects made ambulatory visits to the study centre at scheduled intervals. After collecting the last PK sample between periods and after the period II (72 hours post-dose) an accelerated elimination procedure using cholestyramine $8 \mathrm{~g}$ tid or $4 \mathrm{~g}$ tid, or activated charcoal, as recommended by the originator (SANOFI, Winthrop Industry) was done. The reason for the accelerated drug elimination procedure after period I was to get plasma levels rapidly under the Lowest Limit of Quantification (LLOQ) before starting period II and to increase safety of the subjects participating to the study. It is documented that after a single dose of $14 \mathrm{mg}$ of teriflunomide the plasma levels can be above $0.02 \mathrm{mcg} / \mathrm{ml}$ for an average of 8 months, and these levels are related to fetus malformation. After this accelerated elimination procedure was finalized, blood sample/s for quantification of teriflunomide were drawn and only after plasma concentrations reached levels below LLOQ the restriction with regard to procreation was lifted. The wash out period for this study was 5 weeks.

\section{Analytical methodology}

The identification and quantification of teriflunomide in plasma were performed by a Liquid Chromatography/Mass Spectrometry (HPLC/MS/MS) method using 13C5- teriflunomide as the internal standard. The analytical method was fully validated in accordance with the last bioanalytical validation guideline by before the start of the analysis of the plasma samples EMA [18]. All procedures were carried out on plasma collected with K2EDTA (the anticoagulant used for volunteers sampling). A simple protein precipitation method was employed for sample extraction and HPLC separations were carried out on reversed phase columns using a composition gradient. The eluent from the HPLC column was introduced in the mass spectrometer, Shimadzu LCMS 8060, atmospheric pressure ionization source without splitting. The method allowed for the selective determination of teriflunomide in a range within $5.000 \mathrm{ng} / \mathrm{ml}$ to $5000.000 \mathrm{ng} / \mathrm{ml}$ from aliquots of just $100 \mu \mathrm{L}$ of plasma.

\section{Pharmacokinetic calculations}

The comparative statistics of the pharmacokinetic (PK) data was performed comparing the TEST vs. REFERENCE formulations in fasting conditions. All PK and statistical calculations were performed using SAS version 9.4 software and in particular the GLM procedures (SAS Institute Inc., Cary, NC-USA). Descriptive statistics was done for all pharmacokinetic parameters (arithmetic mean, harmonic mean, geometric mean, Estandar Error of the Mean (SEM), standard deviation (SD), median, range). The bioavailability comparison was carried out on the following teriflunomide pharmacokinetic parameters: AUC0-72 and $\mathrm{C}_{\max }$ (primary pharmacokinetic parameters). The confidence interval for the ratio of the population medians (TEST/ REFERENCE) was calculated considering a classic (shortest) $90 \%$ standard confidence interval. The two one-sided parametric t-tests according to Schuirmann (ln-transformed values) were applied with the null hypothesis of bioinequivalence at the $5 \%$ significance level. The bioequivalence acceptance interval was set to $80-125 \%$ for AUC072 and $\mathrm{C}_{\max }$ of teriflunomide. In accordance with the guidelines of the European Committee for Medicinal Products for Human Use (CHMP) bioequivalence was concluded if the calculated $90 \%$ confidence interval of the ratios was completely within the accepted bioequivalence range. $\mathrm{T}_{\max }$ (secondary pharmacokinetic parameter) was compared using a non-parametric statistical method: Wilcoxon Signed-Rank Test. The effects of sequence, subject (sequence), period and treatment on teriflunomide AUC0-72 and $\mathrm{C}_{\max }$ were separately evaluated, on log-transformed data, using the ANOVA model implemented by GLM procedure of SAS; as limit of statistical significance, $p<0.05$ was considered and the tests were applied to untransformed data.

\section{Report of adverse events}

For adverse events (AEs) descriptive statistics was performed; a single sample proportion test was applied by groups of treatment for the incidence of subjects having encountered AEs and for the incidence of AEs. 
Citation: Sevinsky E, Silvestro L, Neatu A, Roffé C, Rizea-Savu S (2018) Pharmacokinetic Bioequivalence Study of Two Formulations of Teriflunomide in Healthy Male Volunteers under Fasting Conditions. J Bioequiv Availab 10: 43-47. 376. doi: 10.4172/0975-0851.1000376

\section{Results}

The main anthropo-morphometric data of the sample under study are summarized in Table 1.

\section{Data analysis}

Based on plasma concentration results obtained in the 24 participants (Figure $1 \mathrm{a}$ and $\mathrm{b}$ ), pharmacokinetic parameters were estimated for teriflunomide (Table 2). Latin-square ANOVA for teriflunomide showed no significant effect of sequence and period for both primary parameters while a treatment effect was observed only for AUC0-72. Subject within sequences effect was found for both primary parameters. Subject effects are frequently observed due to interindividual variations, without affecting the validity of the study. The intra-subject variability coefficients, registered for teriflunomide, were $3.814 \%$ for AUC $0-72$ and $9.296 \%$ for $\mathrm{C}_{\max }$. The very low intra-subject variability found for AUC 0-72 was the reason while a treatment effect was observed for this parameter in the Latin-square ANOVA evaluation despite the Test/Reference ratio was very close $(103.115 \%$ as in Table 2). For bioequivalence assessment, the statistical tests used to compare AUC0-72 and $\mathrm{C}_{\max }$ did not show statistically significant differences depending on the Teriflunomide preparation. The $90 \%$ confidence intervals of AUC0-72 and $\mathrm{C}_{\max }$ ratios of teriflunomide were completely within the accepted bioequivalence range $80.00-125.00 \%$, thus permitting to conclude for bioequivalence of teriflunomide;

\begin{tabular}{|c|c|c|c|c|}
\hline & Mean & SD & Min & Max \\
\hline Age (years) & 30.38 & 9.36 & 18 & 51 \\
\hline Body weight (Kg) & 72.21 & 9.44 & 55 & 93 \\
\hline Height (cm) & 175.83 & 7.07 & 165 & 190 \\
\hline BMI & 23.31 & 2.82 & 19.7 & 29.1 \\
\hline
\end{tabular}

Table 1: Main features of the sample included in the pharmacokinetic study of two formulations of teriflunomide ( $n=24$ Caucasian men).
Schuirmann tests applied also sustained the results. The statistical analysis of the pharmacokinetic data obtained in this study showed that the Teriflunomide formulations TEST and REFERENCE were bioequivalent regarding the rate $\left(\mathrm{C}_{\max }\right)$ and the extent of absorption (AUC0-72), in fasting conditions. Bioequivalent results are shown in (Table 2). All endpoints have proved bioequivalence and acceptability for the interchangeability of both formulas within the 80-125 range of $90 \%$ CI.

\section{Safety evaluation}

No adverse events occurred in the present study except for the cholestyramine intolerance symptoms (nausea, vomiting, diarrhoea, bloating and abdominal pain) and for the haemoglobin, hematocrit and red blood cells values found outside the normal range at follow-up for one subject which was considered not clinically significant.

\section{Conclusion}

The analysis of the results obtained for the safety parameters considered, permitted to conclude that the two teriflunomide treatments (TEST and REFERENCE), administered in single dose, orally, to Caucasian male healthy volunteers under fasting conditions were very well tolerated by all the subjects enrolled in the study.The $90 \%$ confidence intervals of AUC0-72 and $\mathrm{C}_{\max }$ ratios of teriflunomide were completely within the accepted bioequivalence range 80.00 $125.00 \%$, thus permitting to conclude for bioequivalence for teriflunomide; Schuirmann tests applied also sustained the results. It is noteworthy that the bioequivalence parameters were also adequate when a narrow therapeutic index drug approach was applied. This approach is often employed for critical drugs like immunosuppressant (i.e. cyclosporine and tacrolimus). In conclusion the statistical analysis of the pharmacokinetic data obtained in this study showed that the Teriflunomide formulations: TERFLIMIDA coated tablets $14 \mathrm{mg} /$ Tuteur S.A.C.I.F.I.A, Buenos Aires, Argentina and TEST Aubagio

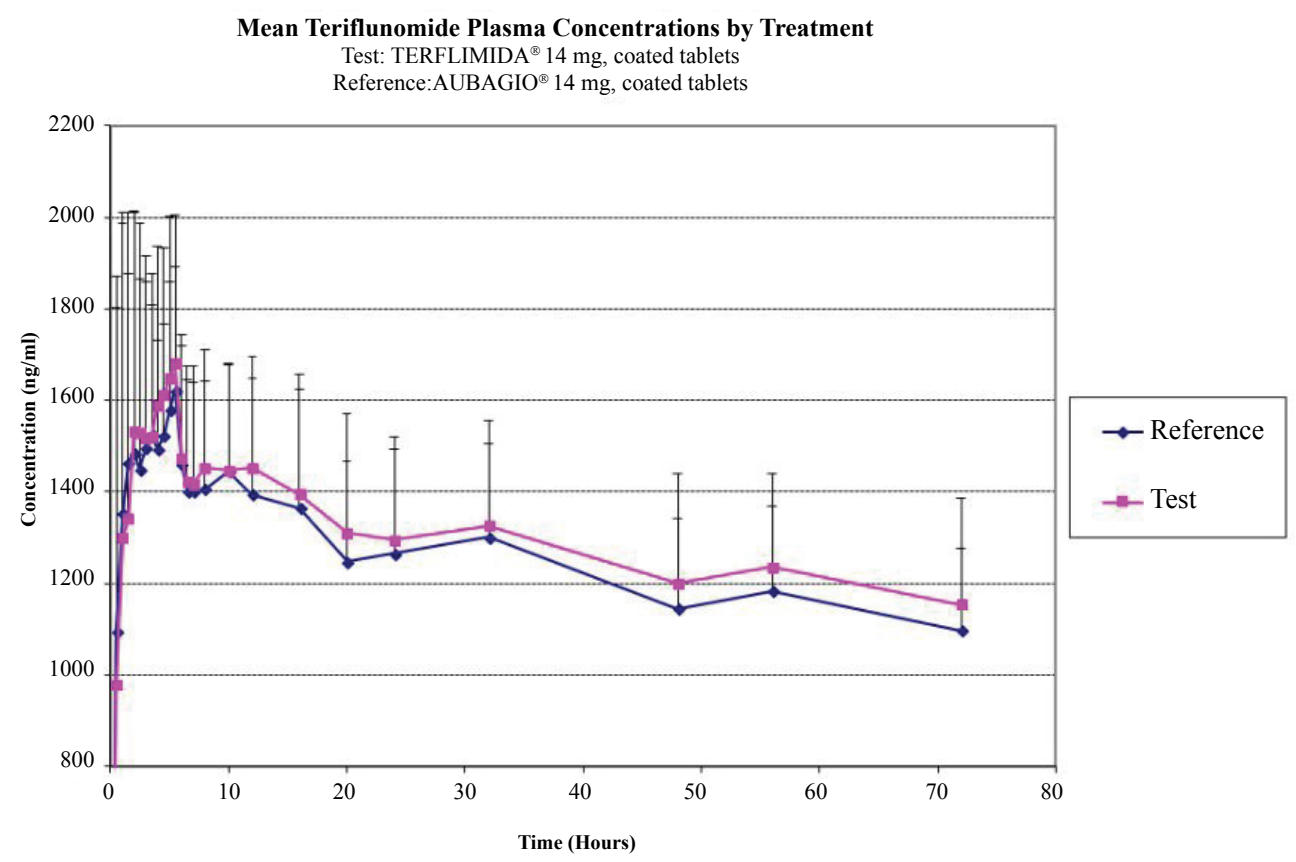

Figure 1a: Mean (+SD) teriflunomide plasma concentration versus time profiles up to $72 \mathrm{~h}$ after test and reference treatments (linear scale) ( $\mathrm{n}=24)$. Abbreviation: SD, standard deviation. 
Citation: Sevinsky E, Silvestro L, Neatu A, Roffé C, Rizea-Savu S (2018) Pharmacokinetic Bioequivalence Study of Two Formulations of Teriflunomide in Healthy Male Volunteers under Fasting Conditions. J Bioequiv Availab 10: 43-47. 376. doi: 10.4172/0975-0851.1000376

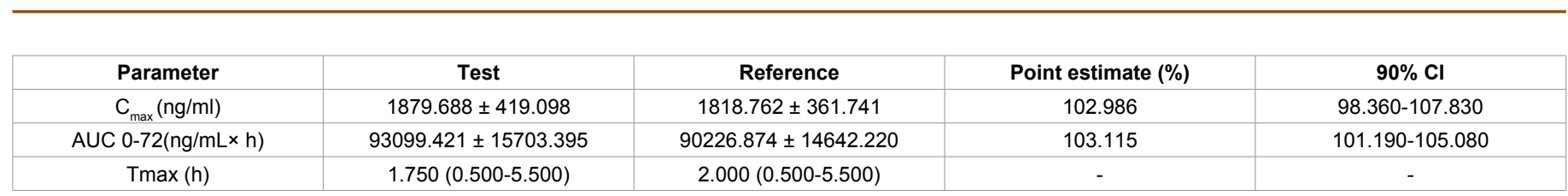

Table 2: Mean plasma teriflunomide pharmacokinetic parameters $(n=24)$.

Notes: Values are mean \pm standard deviation (SD), except for $\mathrm{T}_{\max }$, median (range). Point estimate Test/Reference ratio of geometric means. Test Teriflunomide 14 mg (Terflimida $^{\circledR}$ ); Reference Teriflunomide $14 \mathrm{mg}\left(\right.$ Aubagio $^{\circledR}$ )

Abbreviations:AUC 0-72, area under the curve from 0 to $72 \mathrm{~h}$; $\mathrm{Cl}$, confidence interval; $\mathrm{C}_{\max }$, maximum plasma concentration; $\mathrm{T}_{\text {max }}$, time to maximum plasma concentration.

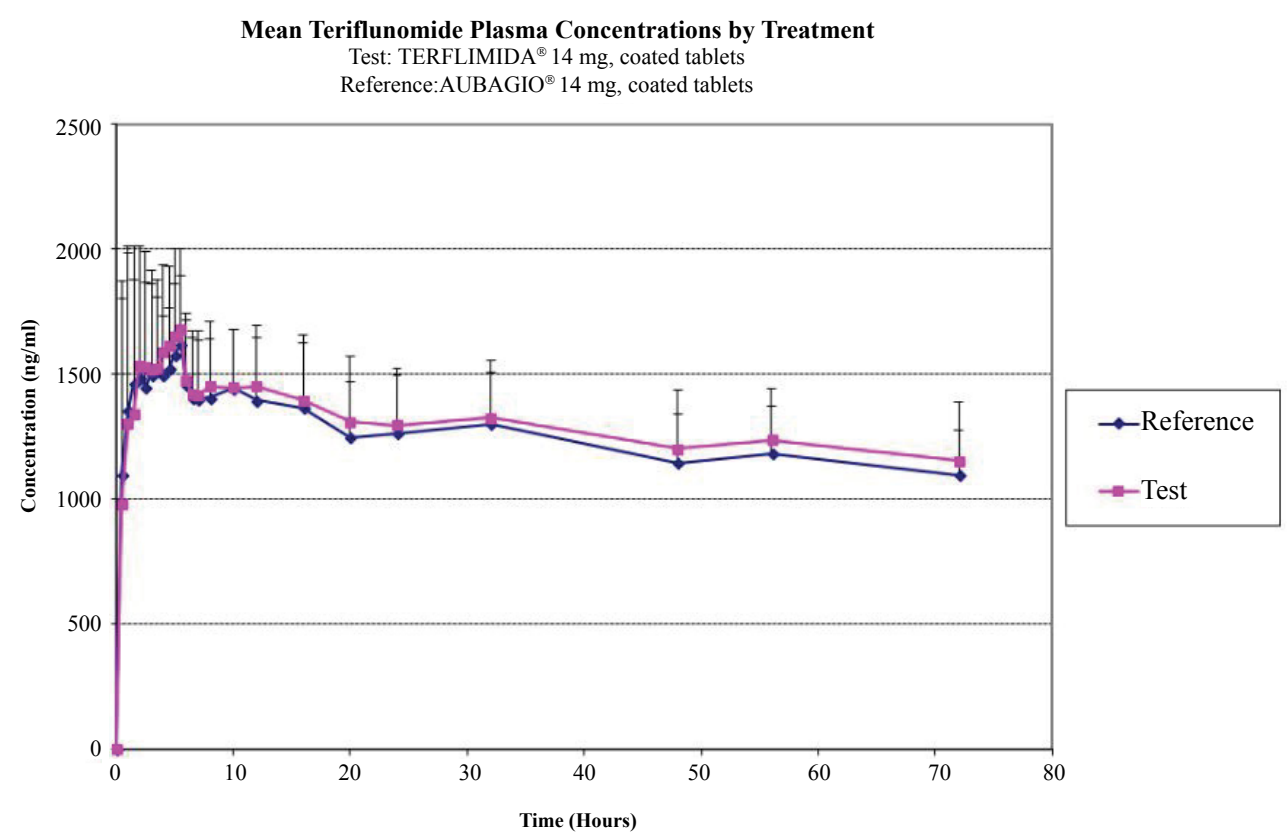

Figure 1b: Mean (+SD) teriflunomide plasma concentration versus time profiles up to $72 \mathrm{~h}$ after test and reference treatments (different scale) ( $\mathrm{n}=24)$.

$14 \mathrm{mg}$ coated tablets manufactured by Sanofi Winthrop Ind., France and imported / distributed by Genzymede Argentina S.A., Argentina (REFERENCE), are bioequivalent regarding the rate $\left(\mathrm{C}_{\max }\right)$ and the extent of absorption (AUC0-72) for teriflunomide, in fasting conditions also applying the most stringent bioequivalence rules. The application of AUC0-72 instead of the more classical AUC0-t permitted to employ a method for accelerated elimination of teriflunomide shortening the total time of the study of several months with a significant safety advantage for the study volunteers.

\section{Acknowledgment}

This study was sponsored by TUTEUR S.A.C.I.F.I.A laboratory. The bioequivalence study and the bioequivalence analysis were carried out by 3S-Pharmacological Consultation \& Research GmbH, Harpstedt, Germany.

\section{References}

1. Katsara M, Matsoukas J, Deraos G, Apostolopoulos V (2008) Towards immunotherapeutic drugs and vaccines against multiple sclerosis. Acta Biochim Biophys 40: 636-642.

2. Lublin FD, Reingold SC (1996) Defining the clinical course of multiple sclerosis: Results of an international survey. Neurol 46: 907-911.

3. Eckstein C, Bhatti MT (2016) Currently approved and emerging oral therapies in multiple sclerosis: An update for the ophthalmologist. Surv. Ophthalmol 61: 318-332.

4. Diebold M, Derfuss T (2016) Immunological treatment of multiple sclerosis. Semin Hematol 53: S54-S57.
5. Katsara M, Minigo G, Plebanski M, Apostolopoulos V (2008) The good, the bad and the ugly: How altered peptide ligands modulate immunity. Expert Opin Biol Ther 8: 1873-1884.

6. Katsara M, Tselios T, Deraos S, Deraos G, Matsoukas MT, et al. (2006) Round and round we go: Cyclic peptides in disease. Curr Med Chem 13: 2221-2232.

7. Palmer AM (2010) Teriflunomide, an inhibitor of dihydroorotate dehydrogenase for the potential oral treatment of multiple sclerosis. Curr Opin Investig Drugs 11: 1313-1323.

8. Korn T, Magnus T, Toyka K, Jung S (2004) Modulation of effector cell functions in experimental autoimmune encephalomyelitis by leflunomide-mechanisms independent of pyrimidine depletion. J Leukoc Biol 76: 950-960.

9. O’Connor P, Wolinsky JS, Confavreux C, Comi G, Kappos L, et al. (2011) Randomized trial of oral teriflunomide for relapsing multiple sclerosis. $\mathrm{N} \mathrm{Engl} \mathrm{J}$ Med 365: 1293-1303.

10. Sanvito L, Constantinescu CS, Gran B (2011) Novel therapeutic approaches to autoimmune demyelinating disorders. Curr Pharm Des 17: 3191-3201.

11. Yeh EA (2011) Current therapeutic options in pediatric multiple sclerosis. Curr Treat Options Neurol 13: 544-559.

12. O'Connor PW, Li D, Freedman MS, Bar-Or A, Rice GPA, et al. (2006) A phase ii study of the safety and efficacy of teriflunomide in multiple sclerosis with relapses. Neurology 66: 894-900.

13. Confavreux C, O'Connor P, Comi G, Freedman MS, Miller AE, et al. (2014) Oral teriflunomide for patients with relapsing multiple sclerosis (tower): A randomised, double-blind, placebo-controlled, phase 3 trial. Lancet Neurol 13: 247-256.

14. ANMAT Provision No (2881/16). 
Citation: Sevinsky E, Silvestro L, Neatu A, Roffé C, Rizea-Savu S (2018) Pharmacokinetic Bioequivalence Study of Two Formulations of Teriflunomide in Healthy Male Volunteers under Fasting Conditions. J Bioequiv Availab 10: 43-47. 376. doi: 10.4172/0975-0851.1000376

15. Lim YJ, Shim EJ, Kim HS, Oh M, Shon JH, et al. (2013) Pharmacokinetics and bioequivalence evaluation of leflunomide tablets in Korean healthy volunteers. Drug Res (Stuttg) 63: 633-638.

16. Agarwal S, Das A, Ghosh D, Sarkar AK, Chattaraj TK, et al. (2012) Comparative bioequivalence study of leflunomide tablets in Indian healthy volunteers. Arzneimittelforschung 62: 145-8.
17. Parekh JM, Vaghela RN, Sutariya DK, Sanyal M, Yadav M, et al. (2010) Chromatographic separation and sensitive determination of teriflunomide, an active metabolite of leflunomide in human plasma by liquid chromatographytandem mass spectrometry. J Chromatogr B Analyt Technol Biomed Life Sci 878: $2217-2225$

18. Committee for Medicinal Products for Human Use (CHMP): Guideline on validation of bioanalytical methods (2009) European Medicines Agency. 